\title{
A Wide-Beam Scanning Mode for Near-Space Passive SARs
}

\author{
Peng Zhou, Peng Ren, and Yongshou Dai \\ College of Information and Control Engineering, China University of Petroleum, Qingdao 266580, China \\ Correspondence should be addressed to Peng Zhou, hdpuzp@163.com
}

Received 13 September 2012; Accepted 20 November 2012

Academic Editor: Gui Gao

Copyright () 2012 Peng Zhou et al. This is an open access article distributed under the Creative Commons Attribution License, which permits unrestricted use, distribution, and reproduction in any medium, provided the original work is properly cited.

\begin{abstract}
A near-space passive SAR consists of a passive radar receiver placed on a near-space vehicle and another existing radar on a satellite serving as an illuminator. In this paper, we present a wide-beam scanning mode for near-space passive SARs for the purpose of increasing the azimuth scene extension. The receiving distance of a near-space SAR is much shorter than that of a satellitebased SAR, and the synthetic aperture time for a near-space passive SAR is not longer than the time interval during which a target transverses the azimuth beam of a transmitter. We design our novel mode by spreading the azimuth beam of a receiver to match the azimuth footprint of the transmitter. We also synchronize the azimuth beam of the receiver with that of the satellite within seconds during the beam scanning to further increase the scene extension. Furthermore, we formulate the azimuth scene extension and the footprint overlapping time for near-space passive SARs in terms of the possible locations of the involved satellites and receivers. Experimental simulations demonstrate the effectiveness of our proposed mode, especially revealing that the widebeam scanning mode improves the azimuth scene extension and footprint overlapping time over the wide-beam mode, without decreasing azimuth resolution.
\end{abstract}

\section{Introduction}

In the study of astronautics and aeronautics, the space between the traditional realms of satellites and typical airplanes is commonly referred to as the near-space, which is quantitatively defined as the atmospheric region from about 20 kilometers to 100 kilometers above the Earth's surface [1]. Since the near-space is above the troposphere, there are no atmospheric obstacles (e.g., clouds, thunderstorms, and precipitation) against remote detections or long-term communications. Therefore, the near-space exhibits great potentials for extensive applications to space attack and defense systems.

Near-space passive SARs [2] are a new type of synthetic aperture radar (SAR). The receiver of a near-space passive SAR is placed on a near-space vehicle. On the other hand, it employs another existing radar on a satellite as the illuminator. Near-space passive SARs are considered as a typical unit with asymmetric structure in the future sensor web [3] because the altitude and velocity of a near-space vehicle is very different from those of a satellite.
Spaceborne/airborne hybrid bistatic SAR [4-7], whose transmitter is spaceborne and receiver is placed on an aircraft, has a resemblance to a near-space passive SAR. However, the latter has two advantages over the former, that is, (1) wider imaging swaths introduced by the greater flying altitude of a receiver platform and (2) greater flexibility induced by the variability of the velocity of a receiver platform, ranging from approximately 5 to 3000 meters per second.

On the other hand, one common feature for near-space passive SARs and spaceborne/airborne hybrid bistatic SARs is that there is a great difference between the velocity of the transmitter platform and that of the receiver platform with regard to a point target. As a result, neither of the two classes of SARs can work on the classic strip-map mode [8]. In the research literature of spaceborne/airborne hybrid bistatic SARs, a double sliding spotlight mode has been proposed to increase the azimuth scene extension, depending on the beam steering on both sides [9]. However, this mode is not applicable to systems using sources of opportunity. To address this shortcoming, a wide-beam mode has been 
studied [10] for noncooperative systems, in accordance with the fact that the receiving distance of a hybrid bistatic SAR is far shorter than that of a monostatic spaceborne SAR. In this paper, we present a wide-beam scanning mode, which enables a hybrid bistatic SAR to further increase the azimuth scene extension. The proposed mode can be used for bistatic SARs with asymmetric structure. In our work, simulations and evaluations will be made for testing the mode for nearspace passive SARs.

\section{Wide-Beam Scanning Mode}

2.1. Overview. The receiving distance of a near-space SAR is much shorter than that of a satellite-based SAR. Furthermore, its synthetic aperture time is shorter or equal to the time interval during which a target traverses the azimuth beam of the transmitter. Based on these two properties of near-space passive SARs, we design a novel mode by spreading the azimuth beam of a near-space SAR to increase the azimuth scene extension. Furthermore, we synchronize the azimuth beam of the receiver with that of the satellite within seconds during the beam scanning to further increase the scene extension. Note that here we assume the receivers are equipped with phased array antennas to perform the beam scanning. We refer to the proposed mode as the wide-beam scanning mode. This mode allows the footprint velocity of a receiver in azimuth to be increased to that of a satellite footprint, provided that the azimuth footprint of the receiver is spread to the same width as that of the azimuth footprint of the transmitter. Under this condition, the synthetic aperture time achieves its maximum value.

Figure 1 illustrates the spatial layout of the proposed wide-beam scanning mode. At the time $t=t_{\text {start }}$, the receiver's beam starts scanning. The angle $\Lambda_{\max }$ is the maximum steering angle of the beam of the receiver. When $t=0$, the satellite and the near-space vehicle pass by the scene center simultaneously and the receiver is side looking. At the time $t=t_{\text {end }}$, the scanning of the beam of the receiver comes to an end. The length $L_{\text {scene }}$ is the achieved azimuth scene extension.

2.2. SNR for a Near-Space Passive SAR. The radar equation for a bistatic SAR [11] is

$$
P_{r}=\frac{P_{t} \lambda^{2} G_{t} G_{r} \sigma_{b}^{0} A_{\mathrm{res}, b}}{(4 \pi)^{3} R_{t}^{2} R_{r}^{2}} .
$$

Here $P_{t}$ and $P_{r}$ are the transmitted and received powers, respectively. $G_{t}$ and $G_{r}$ are the transmitting and receiving gains, respectively. $R_{t}$ and $R_{r}$ are the distances from the transmitter and the receiver to the target, respectively. $\lambda$ is the signal wavelength, $\sigma_{b}^{0}$ is the bistatic normalized radar cross section, and $A_{\text {res, } b}$ is the area of the resolution cell.

For simplicity, we assume that the satellite and the nearspace vehicle move in parallel flight paths and towards the

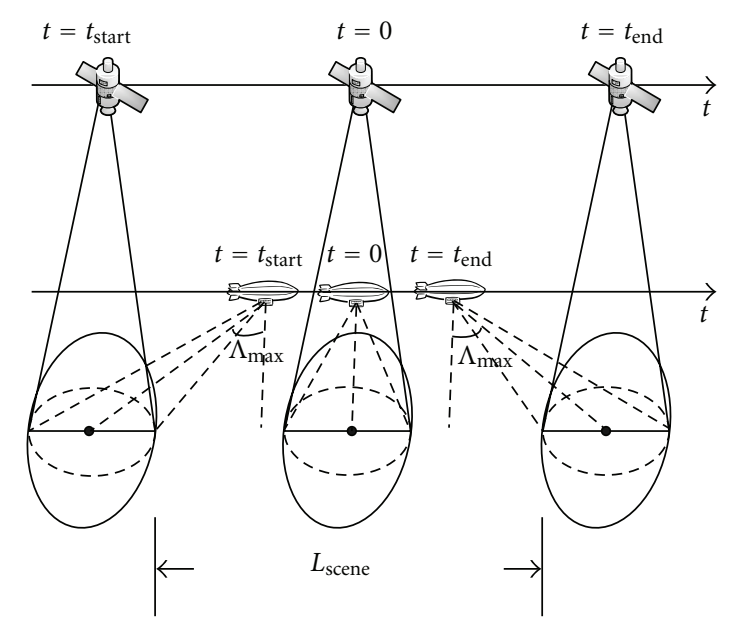

Figure 1: Spatial layout for the wide-beam scanning mode.

same direction. In this scenario, the ground-range and the azimuth resolution can be computed as $[11,12]$

$$
\begin{gathered}
\rho_{\mathrm{gr}, b}=\frac{C}{2 B \cos (\beta / 2) \sin \eta_{b}}, \\
\rho_{\mathrm{az}, b}=\frac{\lambda}{V_{t} / R_{t}+V_{r} / R_{r}} \cdot \frac{1}{\xi_{\text {int }, b}} .
\end{gathered}
$$

Here $\rho_{\mathrm{gr}, b}$ and $\rho_{\mathrm{az}, b}$ are the ground-range and the azimuth resolution, respectively. $V_{t}$ and $V_{r}$ are the flying speeds of the transmitter and the receiver platform respectively, $C$ is the speed of light, $B$ is the bandwidth of the transmitted signal, $\beta$ is the bistatic angle, $\eta_{b}$ is the incidence angle of the bistatic bisector direction, and $\xi_{\text {int }, b}$ is the synthetic aperture time. The area of the resolution cell is the product of the groundrange and the azimuth resolution.

Substituting (2) into (1) and taking the range and azimuth compression gains into consideration, we have

$$
\begin{aligned}
\mathrm{SNR}_{b}= & \frac{P_{t} \lambda^{3} G_{t} G_{r} \sigma_{b}^{0} \eta}{(4 \pi)^{3} R_{t}^{2} R_{r}^{2} k T_{r} F_{r} L_{b}} \\
& \cdot\left(\frac{1}{V_{t} / R_{t}+V_{r} / R_{r}} \cdot \frac{C}{2 B \cos (\beta / 2) \sin \eta_{b}}\right),
\end{aligned}
$$

where $\mathrm{SNR}_{b}$ is the signal-to-noise ratio after range and azimuth compression, $\eta$ is the duty cycle, $k$ is the Boltzmann constant, $T_{r}$ is the receiver noise temperature, $F_{r}$ is the receiver noise figure, and $L_{b}$ is the system loss factor.

To make the beam coverage in azimuth identical, we set the azimuth antenna length of the near-space vehicle to

$$
l_{\mathrm{az}, r}=\frac{R_{r}}{R_{t}} \cdot \frac{l_{\mathrm{az}, t}}{\cos \Lambda_{r}} .
$$

Here $l_{\mathrm{az}, t}$ and $l_{\mathrm{az}, r}$ are the azimuth antenna length of the satellite and the near-space vehicle, respectively. $\Lambda_{r}$ is the azimuth scanning angle of the receiving antenna, which is varying in the scanning process. The presence of $\Lambda_{r}$ is caused 
by the beam spread effect during the beam scanning process. The SNR under this condition is given by

$$
\begin{aligned}
\mathrm{SNR}_{b}= & \frac{P_{t} \rho_{t} \rho_{r} l_{\mathrm{az}, t}^{2} l_{\mathrm{el}, t} l_{\mathrm{el}, r} \sigma_{b}^{0} \eta}{4 \pi \lambda R_{t}^{3} R_{r} k T_{r} F_{r} L_{b}} \\
& \cdot\left(\frac{1}{V_{t} / R_{t}+V_{r} / R_{r}} \cdot \frac{C}{2 B \cos (\beta / 2) \sin \eta_{b}}\right) .
\end{aligned}
$$

Here $\rho_{t}$ and $\rho_{r}$ are the aperture efficiencies of the transmitting and receiving antennas, respectively. $l_{\mathrm{el}, t}$ and $l_{\mathrm{el}, r}$ are the elevational antenna lengths of the satellite and the near-space vehicle, respectively. Note that there is no parameter $\Lambda_{r}$ in (5) due to the SNR loss caused by the azimuth scanning of the receiving antenna. For a monostatic spaceborne SAR, we reformulate (5) as follows:

$$
\mathrm{SNR}_{t}=\frac{P_{t} \rho_{t}^{2} l_{\mathrm{a}, t}^{2} l_{\mathrm{el}, t}^{2} \sigma^{0} \eta}{4 \pi \lambda R_{t}^{4} k T_{t} F_{t} L_{t}} \cdot\left(\frac{R_{t}}{2 V_{t}} \cdot \frac{C}{2 B \sin \eta_{t}}\right)
$$

where $\mathrm{SNR}_{t}$ is the SNR after compression for a monostatic spaceborne SAR, $\sigma^{0}$ is the monostatic normalized radar cross section, and $\eta_{t}$ is the incidence angle in a monostatic spaceborne SAR. Additionally, $T_{t}, F_{t}, L_{t}$ are the receiver noise temperature, the receiver noise figure, and the system loss factor in a monostatic spaceborne SAR, respectively. Dividing (5) by (6), we have

$$
\begin{aligned}
\frac{\mathrm{SNR}_{b}}{\mathrm{SNR}_{t}} & =\frac{\rho_{r} \sigma_{b}^{0} T_{t} F_{t} L_{t} \sin \eta_{t}}{\rho_{t} \sigma^{0} T_{r} F_{r} L_{b} \cos (\beta / 2) \sin \eta_{b}} \cdot \frac{l_{\mathrm{el}, r}}{l_{\mathrm{e}, t, t}} \cdot \frac{2}{R_{r} / R_{t}+V_{r} / V_{t}} \\
& =K \cdot \frac{l_{\mathrm{el}, r}}{l_{\mathrm{el}, t}} \cdot \frac{2}{R_{r} / R_{t}+V_{r} / V_{t}},
\end{aligned}
$$

where $K$ is a variable for brevity, whose value is around 1 .

We then make an estimate of the SNR ratio. We assume that an X-band transmitter is placed on a LEO satellite flying at approximately $515 \mathrm{~km}$ altitude with $R_{t}=700 \mathrm{~km}$ and a near space receiver is flying at an altitude of $20 \mathrm{~km}$ with $R_{r}=$ $35 \mathrm{~km}$, and $V_{r}=5 \mathrm{~m} / \mathrm{s}, V_{t}=7600 \mathrm{~m} / \mathrm{s}, l_{\mathrm{el}, t}=0.7 \mathrm{~m}, l_{\mathrm{el}, r}=$ $0.08 \mathrm{~m}, K \approx 1$, and the calculated SNR ratio is about 4.5. This ratio that is greater than 1 results from the much shorter receiving distance compared to the transmitting distance and assures the feasibility of the proposed mode. In particular, the receiving beam can be spread by weighting the amplitude and phase of the phased array elements, and thus the ratio can be significantly increased.

2.3. PRF for a Near-Space Passive SAR. For a monostatic spaceborne SAR, the employed PRF satisfies [13]

$$
\left(B_{\mathrm{az}, t} \approx \frac{2 V_{t}}{l_{\mathrm{az}, t}}\right)<\mathrm{PRF}_{t}<\frac{1}{T_{\mathrm{dur}, t}+\tau_{p}},
$$

where $B_{\mathrm{az}, t}, \mathrm{PRF}_{t}, T_{\mathrm{dur}, t}, \tau_{p}$ are the Doppler bandwidth, the employed PRF, the echo duration time, and the pulse width of the transmitted signal in a monostatic spaceborne SAR, respectively.
For a near-space passive SAR the Doppler bandwidth is given by

$$
B_{\mathrm{az}, b} \approx \frac{V_{t}}{l_{\mathrm{az}, t}}+\frac{V_{r}^{2}}{V_{t}^{2}} \cdot \frac{R_{r} R_{t}}{R_{r}^{2}+\left(1-\left(V_{r} / V_{t}\right)\right)^{2} y^{2}} \cdot \frac{V_{t}}{l_{\mathrm{az}, t}},
$$

where $y$ is the coordinate in the azimuth direction and centered at the scene center. The derivation is omitted for the simplicity. According to (9), the Doppler bandwidth achieves its maximum value at the scene center. In this case, the Doppler bandwidth is given as

$$
B_{\mathrm{az}, b, \max } \approx\left(1+\frac{V_{r}^{2}}{V_{t}^{2}} \frac{R_{t}}{R_{r}}\right) \cdot \frac{V_{t}}{l_{\mathrm{az}, t}}
$$

It is clear that if the ratio $\left(V_{r}^{2} / V_{t}^{2}\right) \cdot\left(R_{t} / R_{r}\right)$ is not greater than 1 , the employed PRF for the spaceborne illuminator will not cause the Doppler ambiguity to a near-space passive SAR. In most cases, the above constraint can be satisfied except when the receiver platform moves rapidly, for example, faster than $1500 \mathrm{~m} / \mathrm{s}$ when $R_{t}$ is more than 25 times of $R_{r}$. In this case, some procedures to remove the azimuth spectral folding phenomenon must be taken when designing the imaging algorithm for a near-space passive SAR, and the study of such procedures is another research topic, which is beyond the scope of this work.

Spaceborne/airborne hybrid bistatic SARs and nearspace passive SARs show resemblance in geometric layout of involved elements, because for the SAR systems it is common that the altitude and velocity of a satellite are far greater than those of the corresponding receiver platform. Therefore, in most cases, the echo duration time in a monostatic spaceborne SAR is longer than that in a nearspace passive SAR since the swath is much longer in a monostatic spaceborne SAR, which is essentially similar to the case for a spaceborne/airborne hybrid bistatic SAR. Thus the employed PRF for the spaceborne illuminator will not cause the range ambiguity to a near-space passive SAR. We refer the readers to [10] for a detailed explanation of a spaceborne/airborne hybrid bistatic SAR.

In Summary, if the ratio $\left(V_{r}^{2} / V_{t}^{2}\right) \cdot\left(R_{t} / R_{r}\right)$ is not greater than 1 , the PRF of the illuminator satisfies

$$
B_{\mathrm{az}, b, \max }<\mathrm{PRF}_{t}<\frac{1}{T_{\mathrm{dur}, b}+\tau_{p}},
$$

where $T_{\mathrm{dur}, b}$ is the echo duration time for a near-space passive SAR. On the other hand, when the ratio $\left(V_{r}^{2} / V_{t}^{2}\right) \cdot\left(R_{t} / R_{r}\right)$ is greater than 1 , the azimuth spectral folding phenomenon should be removed. The study of how to remove the spectral folding phenomenon is a whole new nontrivial problem, which will be addressed in our future research.

2.4. Formulation of the Azimuth Scene Extension and the Footprint Overlapping Time. The azimuth scene extension 
TABle 1: Parameters employed in the simulation.

\begin{tabular}{|c|c|c|c|c|c|c|}
\hline Parameters & TerraSAR-X & An $\mathrm{X}$-band receiver & Envisat & A C-band receiver & TerraSAR-L & A L-band receiver \\
\hline Altitude (km) & 515 & 20 & 800 & 20 & 645 & 20 \\
\hline Velocity $(\mathrm{km} / \mathrm{s})$ & 7.6 & 0.005 & 7.45 & 0.005 & 7.53 & 0.005 \\
\hline Incidence angle $\left({ }^{\circ}\right)$ & $20-45$ & $30-50$ & $15-45$ & $30-55$ & $20-45$ & $40-50$ \\
\hline Carrier frequency $(\mathrm{GHz})$ & 9.65 & 9.65 & 5.331 & 5.331 & 1.2575 & 1.2575 \\
\hline Peak power $(\mathrm{kw})$ & 2.26 & - & 2.3 & - & 4 & - \\
\hline $\operatorname{Loss}^{1}(\mathrm{~dB})$ & $2 / 5$ & 3 & $2 / 5$ & 3 & $2 / 5$ & 3 \\
\hline Aperture efficiency & 0.7 & 0.7 & 0.7 & 0.7 & 0.7 & 0.7 \\
\hline normalized radar cross section $(\mathrm{dB})$ & -20 & -20 & -20 & -20 & -30 & -30 \\
\hline Pulse width $(\mu \mathrm{s})$ & 45 & - & 25 & - & 35 & - \\
\hline Noise figure $(\mathrm{dB})$ & 5 & 5 & 5 & 5 & 5 & 5 \\
\hline Noise temperature $(\mathrm{K})$ & 300 & 300 & 300 & 300 & 300 & 300 \\
\hline Bandwidth (MHz) & 150 & 150 & 16 & 16 & 85 & 85 \\
\hline Beamwidth ELV $\left(^{\circ}\right)$ & 2.3 & 18.6 & 2.2 & 12.7 & 4.2 & 21.2 \\
\hline Beamwidth $\mathrm{Az}^{2}\left({ }^{\circ}\right)$ & 0.33 & - & 0.29 & - & 1.1 & - \\
\hline Maximum steering angle $\left({ }^{\circ}\right)$ & - & 30 & - & 30 & - & 40 \\
\hline $\operatorname{PRF}(\mathrm{KHz})$ & 4 & 4 & 2 & 2 & 2 & 2 \\
\hline Operational PRF range $(\mathrm{KHz})$ & $3-6.5$ & - & $1.6-2.5$ & - & $1.5-3$ & - \\
\hline
\end{tabular}

${ }^{1}$ The left of the slash sign is the transmit loss for the near-space passive SAR; the right is the total loss for the monostatic spaceborne SAR.

${ }^{2}$ The azimuth beamwidth of the receiver is calculated by (4).

TABLE 2: Ratio of azimuth scene extension and footprint overlapping time in wide-beam scanning mode to wide-beam mode.

\begin{tabular}{lccc}
\hline Ratio & X-band simulation & C-band simulation & L-band simulation \\
\hline Azimuth scene extension & $5.2-10$ & $3.5-8.3$ & $1.2-2.9$ \\
footprint overlapping time & $3.1-5.5$ & $2.3-4.6$ & $1.1-1.9$ \\
\hline
\end{tabular}

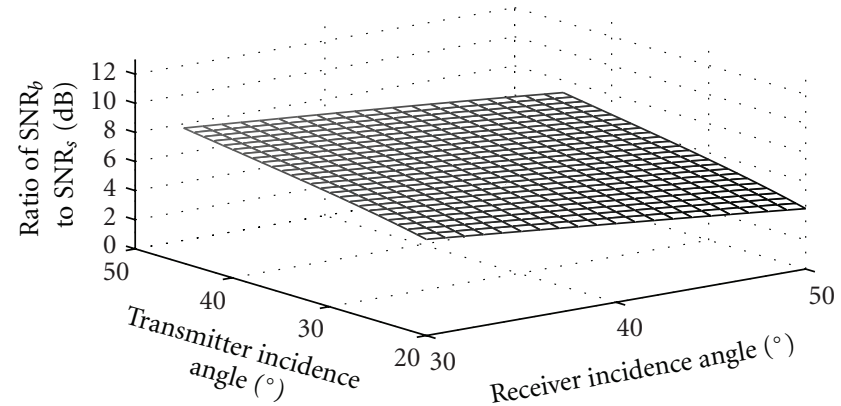

(a) simulation result of $\mathrm{X}$ band

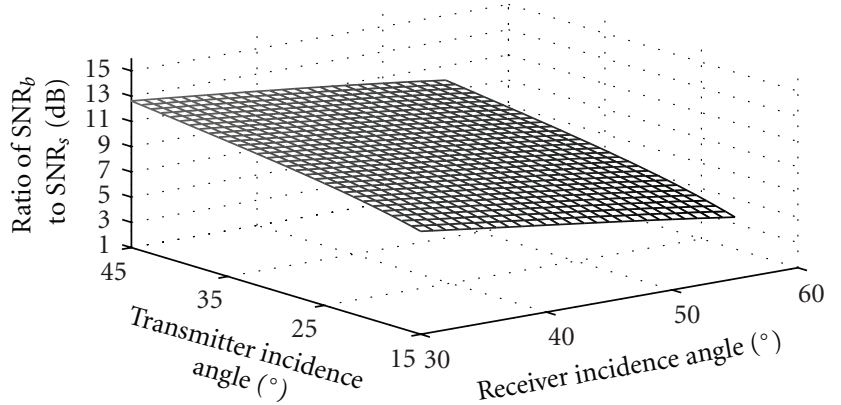

(b) simulation result of $\mathrm{C}$ band

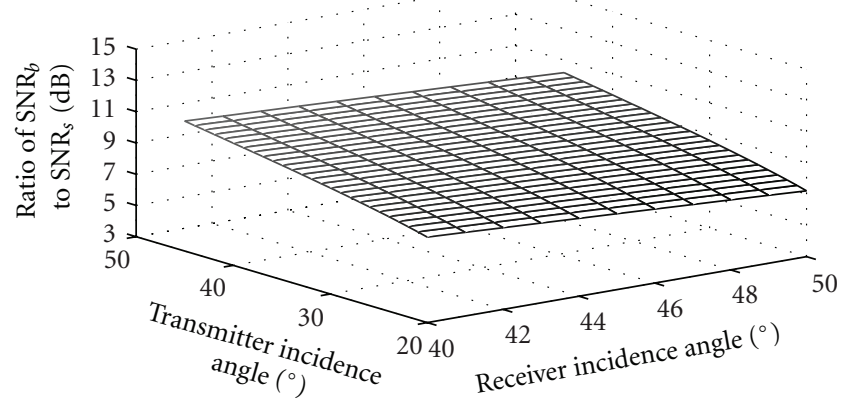

(c) simulation result of $\mathrm{L}$ band

FIGURE 2: Simulation results of SNR ratio. 


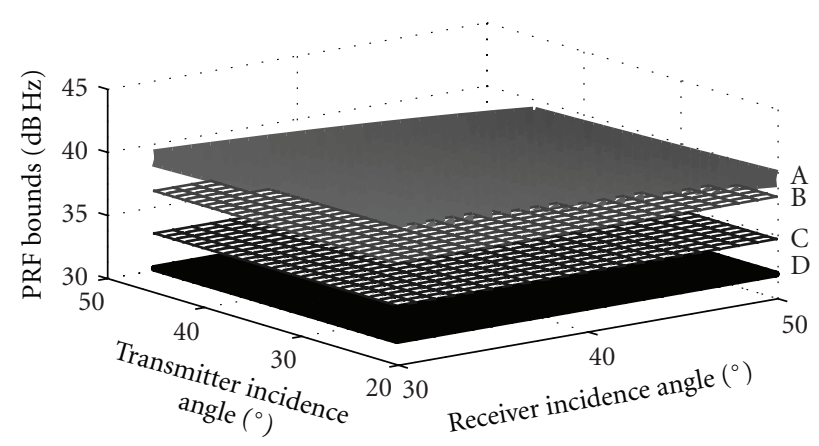

(a) simulation result of $\mathrm{X}$ band

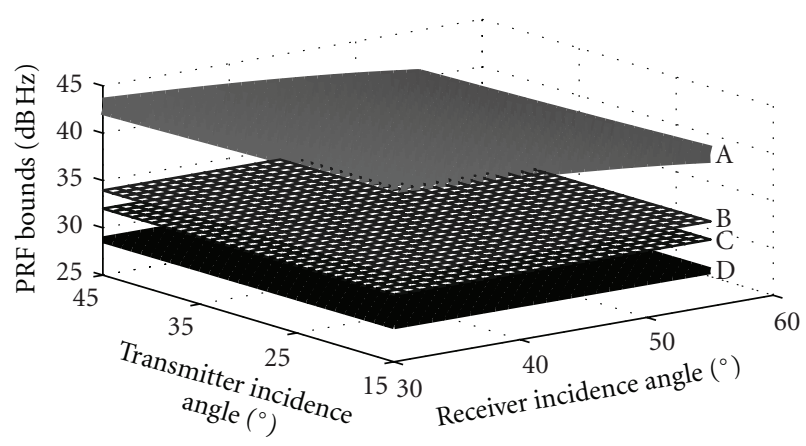

(b) simulation result of $\mathrm{C}$ band

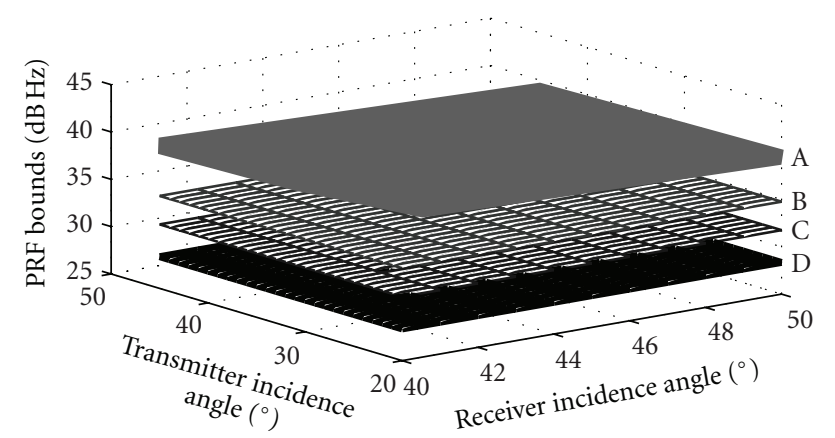

(c) simulation result of $\mathrm{L}$ band

FIGURE 3: Simulation results of PRF bound with $V_{r}=5 \mathrm{~m} / \mathrm{s}$. Curved surface A: the upper bound of the PRF for near-space passive SAR. Curved surface B: the upper bound of the operational PRF range for the corresponding spaceborne illuminator. Curved surface C: the lower bound of the operational PRF range for the corresponding spaceborne illuminator. Curved surface D: the lower bound of the PRF for near-space passive SAR.

$L_{\text {scene }}$ and the footprint overlapping time $t_{\text {overlap }}$ are separately formulated as follows:

$$
\begin{gathered}
L_{\text {scene }}=\frac{V_{r} D_{\mathrm{az}, t}+2 V_{t} R_{r} \cdot \tan \left(\Lambda_{\max }-\left(\theta_{\mathrm{az}, r} / 2\right)\right)}{V_{t}-V_{r}}, \\
t_{\text {overlap }}=\frac{L_{\text {scene }}+D_{\mathrm{az}, t}}{V_{t}} .
\end{gathered}
$$

Here $\theta_{\mathrm{az}, r}$ is the $3-\mathrm{dB}$ beam width of the receiver's beam at the start or end of the scan. $D_{\mathrm{az}, t}$ is the azimuth footprint dimension of the transmitter. The deduction is omitted for brevity.

In our framework, the azimuth resolution discussed in [10] is reformulated as

$$
\rho_{\mathrm{az}, b}=\frac{l_{\mathrm{az}, t}}{1+\left(R_{t} / R_{r}\right) \cdot\left(V_{r} / V_{t}\right)} .
$$

We then make an estimate of their magnitudes using the same parameter values as described in Section 2.2. $\Lambda_{\max }$ is set to $30^{\circ}, l_{\mathrm{az}, t}$ is set to $4 \mathrm{~m}$, and $\lambda$ is set to $0.031 \mathrm{~m}$. The value of $\theta_{\mathrm{az}, r}$ can be computed using (4). In this study, $L_{\text {scene }}$ and $t_{\text {overlap }}$ are approximately $34 \mathrm{~km}$ and $5 \mathrm{~s}$, respectively. In contrast, their values are $4 \mathrm{~km}$ and $1.1 \mathrm{~s}$ under the wide-beam mode presented in [10]. It is clear that with our assumed parameter values, the proposed mode greatly improves $L_{\text {scene }}$ and $t_{\text {overlap }}$ over the wide-beam mode [10]. Nevertheless, the azimuth resolutions for the two modes are the same because of their identical synthetic aperture time.

\section{Simulations}

We conduct simulations to demonstrate the effectiveness of the wide-beam scanning mode. We use the parameters of TerraSAR-X, Envisat, and TerraSAR-L as those of X-, C-, and L-band radar satellites, and these parameters are employed in our simulations. All possible incidence angles, both for the illuminator and for the receiver, are examined. Table 1 lists the parameters used in the simulations. Additionally, the velocity of the receiver is set to 1000,1500 , and $2000 \mathrm{~m} / \mathrm{s}$ separately in the simulations of PRF bounds for different receiver speeds.

We use the parameters in Table 1 to compute the SNR ratio by (7) and show the results in Figure 2. It can be seen that the lowest ratio is greater than $4 \mathrm{~dB}$. This SNR in a near-space passive SAR guarantees acceptable image qualities. We compute the lowest allowable PRF using (10) with relevant parameters. The greatest acceptable values of PRF are also computed according to the geometric properties of the spatial layout of the modes. The results of PRF bounds are depicted in Figure 3, in which the curved surfaces A and $\mathrm{D}$ are the upper and lower bounds of the PRF for the nearspace passive SAR, respectively, and the curved surfaces $B$ 


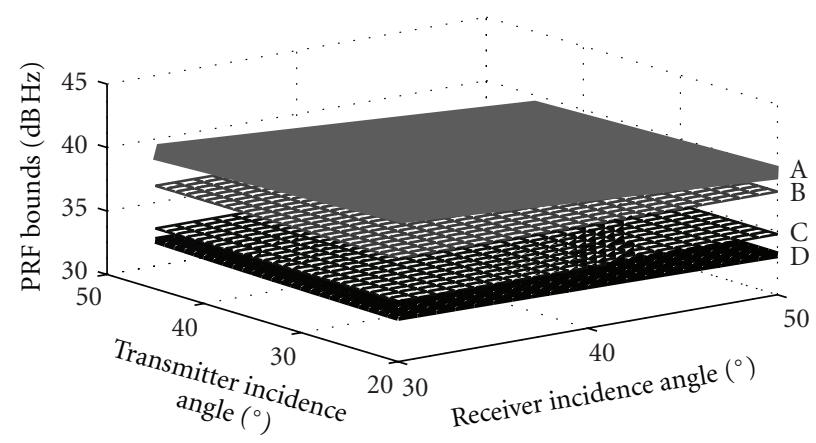

(a) X band simulation result with $V_{r}=1000 \mathrm{~m} / \mathrm{s}$

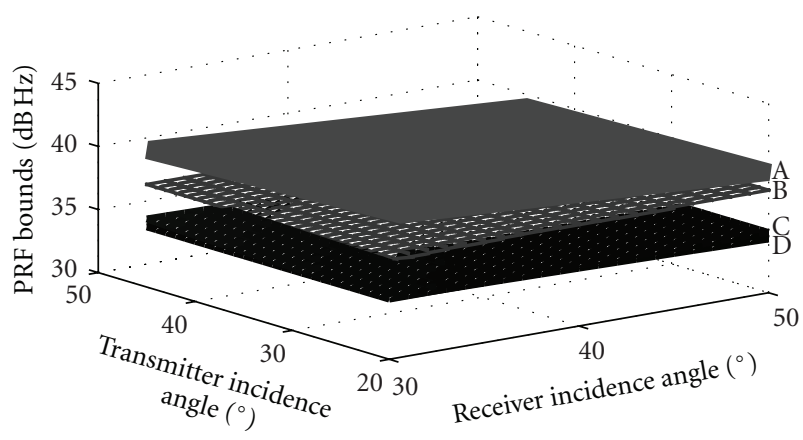

(b) X band simulation result with $V_{r}=1500 \mathrm{~m} / \mathrm{s}$

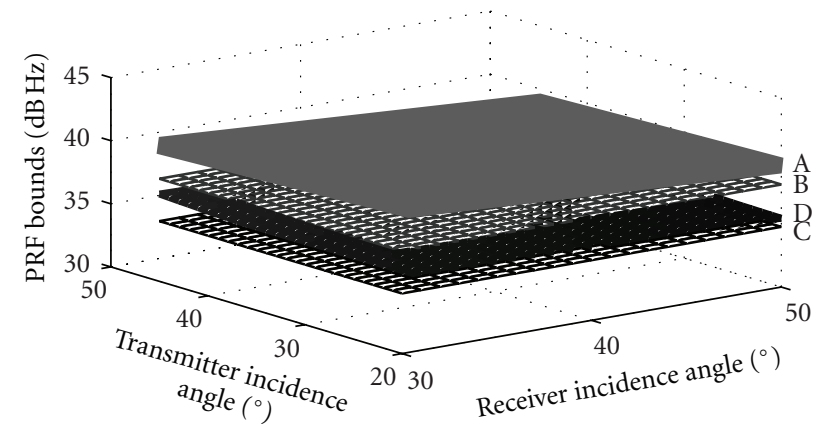

(c) X band simulation result with $V_{r}=2000 \mathrm{~m} / \mathrm{s}$

FIGURE 4: Simulation results of PRF bound with different receiver speed. Curved surface A: the upper bound of the PRF for near-space passive SAR. Curved surface B: the upper bound of the operational PRF range for the corresponding spaceborne illuminator. Curved surface $\mathrm{C}$ : the lower bound of the operational PRF range for the corresponding spaceborne illuminator. Curved surface D: the lower bound of the PRF for near-space passive SAR.

and $\mathrm{C}$ are the upper and lower bounds of the operational PRF ranges for the corresponding spaceborne illuminator, respectively. It is clear that the operational PRF ranges for the spaceborne illuminator are between the acceptable upper and lower bounds for the near-space passive SAR. Note that the receiver velocity is set to $5 \mathrm{~m} / \mathrm{s}$, which is a typical speed of a floater in the near-space. In Section 2, we have observed that the lower bound of the acceptable PRF may be greater than the PRF employed by the spaceborne illuminator when the receiver platform moves rapidly. The third simulation verifies this observation. Figure 4 illustrates the acceptable PRF bound when the receiver's velocity is set to 1000,1500 , and $2000 \mathrm{~m} / \mathrm{s}$ separately. We note that the curved surfaces C and $\mathrm{D}$ nearly overlap when the receiver's velocity is set to $1500 \mathrm{~m} / \mathrm{s}$ and that the curved surface $\mathrm{D}$ is above the surface C when the velocity reaches $2000 \mathrm{~m} / \mathrm{s}$.

Another simulation is performed to compute the ratio of the azimuth scene extension and the footprint overlapping time for the wide-beam scanning mode and the wide-beam mode. The results are listed in Table 2, and it can be seen that the values of the azimuth scene extension and the footprint overlapping time are remarkably improved by using the proposed mode, especially for the $\mathrm{X}$ and $\mathrm{C}$ bands. The reason why the improvement in $\mathrm{L}$ band is relatively small is that the beam width of a receiver at this band is relatively wide due to its relatively long wavelength (see (12)).

\section{Conclusions and Future Work}

We have presented a wide-beam scanning mode for nearspace passive SARs. Theoretical analysis and simulations have shown that the SNR resulting from our method is greater than that for a monostatic spaceborne SAR. This promising result provides the possibility for enabling nearspace passive SARs to image with acceptable qualities. Experimental simulations validate that the azimuth scene extension and the footprint overlapping time in wide-beam scanning mode are greater than the values in wide-beam mode. This improvement from the presented mode does not degrade the azimuth resolution.

In our future work, we will investigate more complicated beam expansion algorithms to further increase the SNR. In other words, we will spread the beam by weighting amplitudes and phases on each array element. We will also study the performance of our new algorithms in improving SNR.

\section{Acknowledgments}

This work is supported by National Natural Science Foundation of China (no. 61105005) and by the Fundamental Research Funds for the Central Universities (no. 27R0905024A). 


\section{References}

[1] W. Q. Wang, "Near-space vehicles: supply a gap between satellites and airplanes for remote sensing," IEEE Aerospace and Electronic Systems Magazine, vol. 26, no. 4, pp. 4-9, 2011.

[2] W.-Q. Wang, J. Cai, and Q. Peng, "Near-space microwave radar remote sensing: potentials and challenge analysis," Remote Sensing, vol. 2, no. 3, pp. 717-739, 2010.

[3] W. Keydel, "Perspectives and visions for future SAR systems," IEE Proceedings, vol. 150, no. 3, pp. 97-103, 2003.

[4] J. H. G. Ender, J. Klare, I. Walterscheid et al., "Bistatic exploration using spaceborne and airborne SAR sensors: a close collaboration between FGAN, ZESS and FOMAAS," in Proceedings of the International Geoscience and Remote Sensing Symposium (IGARSS '06), pp. 1828-1831, Denver, Colo, USA, 2006.

[5] T. Fujimura, H. Totsuka, N. Imai et al., "The bistatic SAR experiment with ALOS/PALSAR and Pi-SAR-L," in Proceedings of the International Geoscience and Remote Sensing Symposium (IGARSS '11), pp. 4221-4224, Vancouver, Canada, 2011.

[6] Z. Liu, J. Yang, X. Zhang, and Y. Pi, "Study on spaceborne/airborne hybrid bistatic SAR image formation in frequency domain," IEEE Geoscience and Remote Sensing Letters, vol. 5, no. 4, pp. 578-582, 2008.

[7] M. Rodriguez-Cassola, S. V. Baumgartner, G. Krieger, and A. Moreira, "Bistatic TerraSAR-X/F-SAR spaceborne-airborne SAR experiment: description, data processing, and results," IEEE Transactions on Geoscience and Remote Sensing, vol. 48, no. 2, pp. 781-794, 2010.

[8] U. Gebhardt, O. Loffeld, H. Nies, S. Knedlik, and J. Ender, "Bistatic airborne/spaceborne hybrid experiment: basic considerations," in Sensors, Systems, and Next-Generation Satellites IX, vol. 5978 of Proceedings of SPIE, Brügge, Belgium, 2005.

[9] I. Walterscheid, T. Espeter, and J. H. G. Ender, "Performance analysis of a hybrid bistatic SAR system operating in the double sliding spotlight mode," in Proceedings of the of International Geoscience and Remote Sensing Symposium (IGARSS '07), pp. 2144-2147, Barcelona, Spain, 2007.

[10] P. Zhou and Y. Pi, "Wide-beam mode and space-time synchronization of antenna footprints in hybrid bistatic SAR systems using sources of opportunity," IEICE Transactions on Communications, vol. E92-B, no. 4, pp. 1308-1317, 2009.

[11] I. Walterscheid, J. Klare, A. R. Brenner, J. H. G. Ender, and O. Loffeld, "Challenges of a bistatic spaceborne/airborne SAR experiment," in Proceedings of the European Conference on Synthetic Aperture Radar (EUSAR '06), Dresden, Germany, 2006.

[12] G. Krieger, H. Fiedler, D. Hounam, and A. Moreira, "Analysis of system concepts for bi- and multi-static SAR missions," in Proceedings of the International Geoscience and Remote Sensing Symposium (IGARSS '03), pp. 770-772, Toulouse, France, July 2003.

[13] J. C. Curlander and R. N. McDonough, Synthetic Aperture Radar-Systems and Signal Processing, John Wiley \& Sons, New York, NY, USA, 1991. 

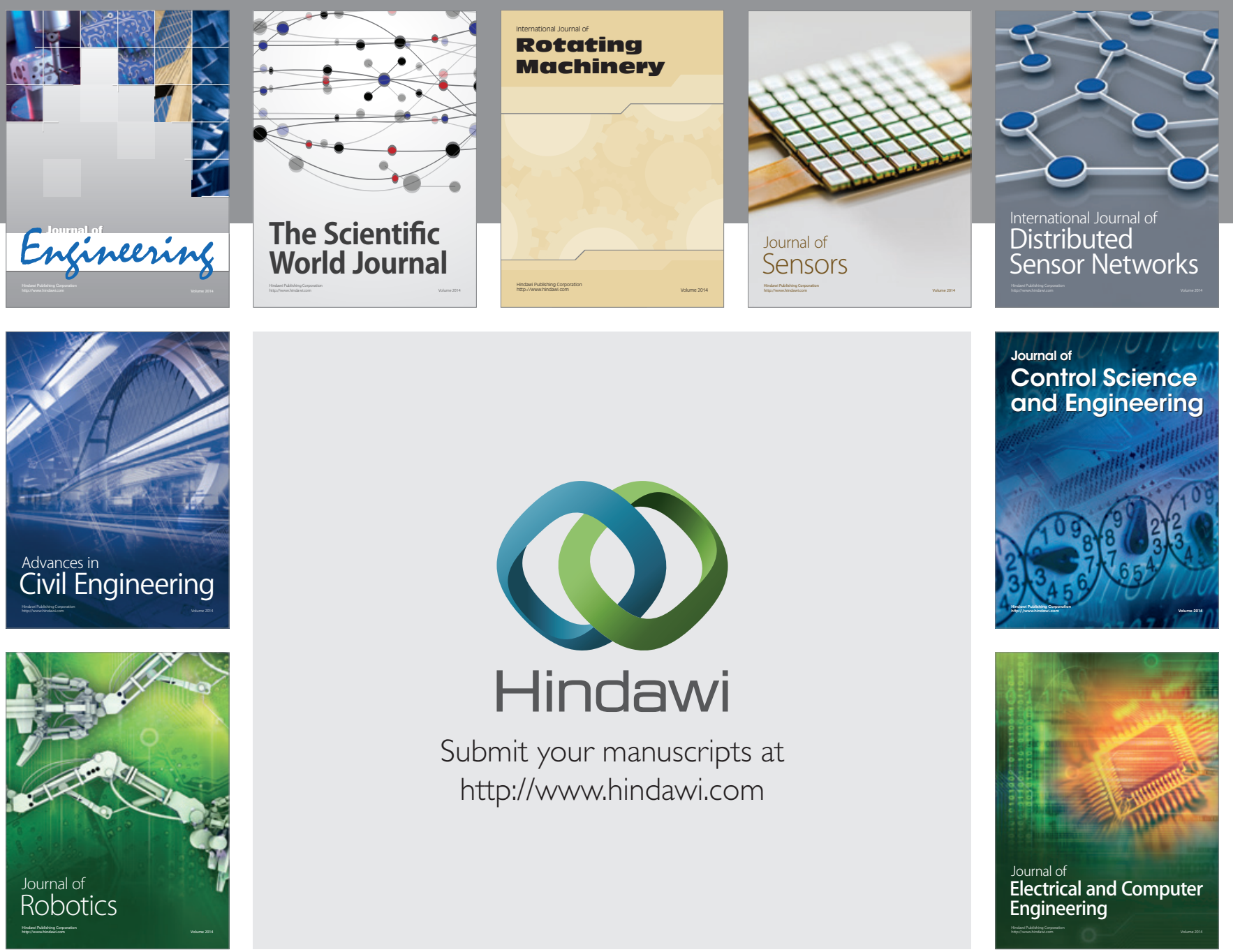

Submit your manuscripts at

http://www.hindawi.com
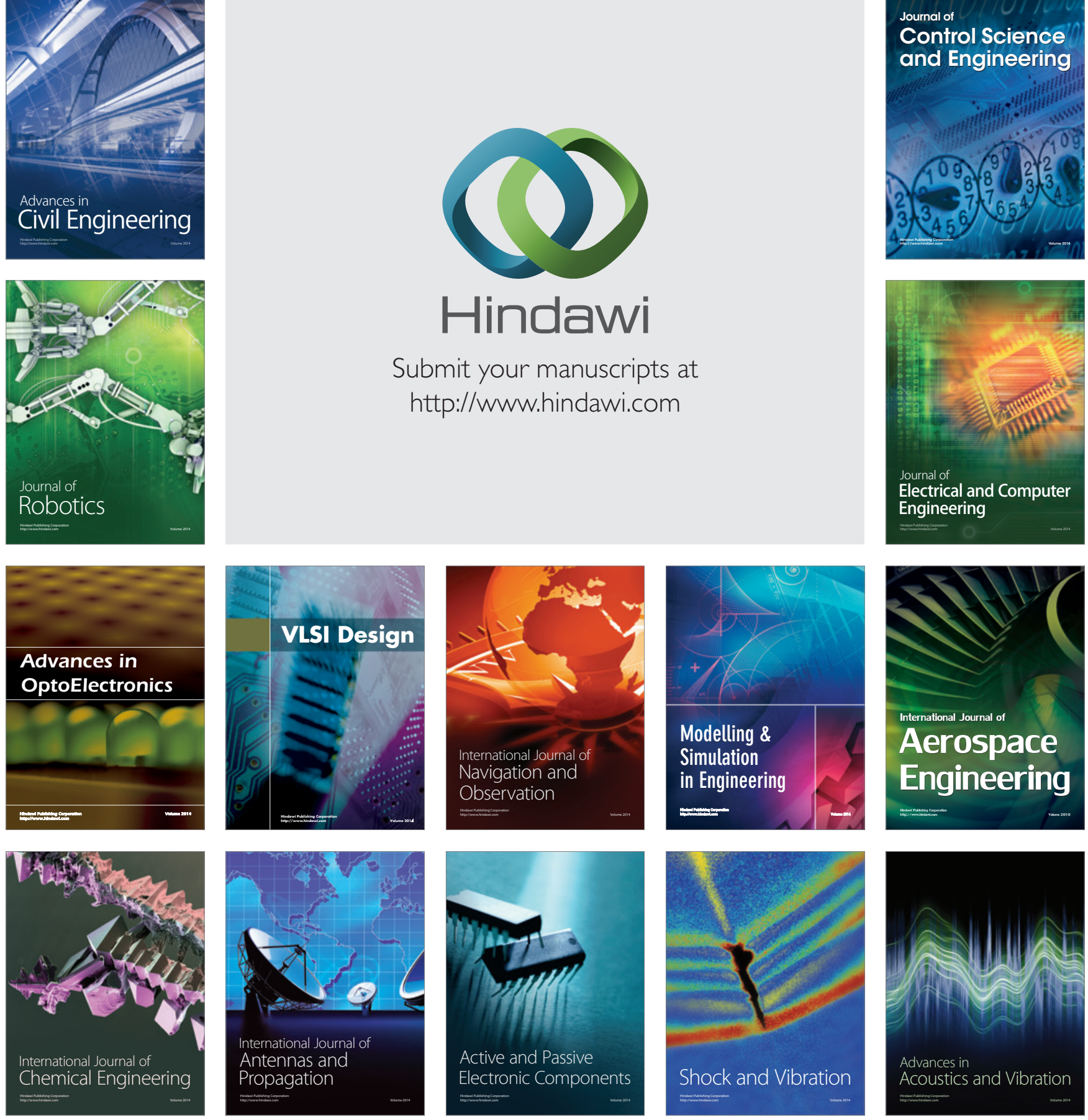